\title{
The Information Geometry of the One-Dimensional Potts Model
}

\author{
B.P. Dolan \\ Department of Mathematical Physics \\ National University of Ireland \\ Maynooth, Ireland \\ D.A. Johnston \\ Dept. of Mathematics \\ Heriot-Watt University \\ Riccarton \\ Edinburgh, EH14 4AS, Scotland \\ and \\ R. Kenna \\ School of Mathematical and Information Sciences \\ Coventry University \\ Coventry, CV1 5FB, England
}

January 20, 2004

\begin{abstract}
In various statistical-mechanical models the introduction of a metric onto the space of parameters (e.g. the temperature variable, $\beta$, and the external field variable, $h$, in the case of spin models) gives an alternative perspective on the phase structure. For the one-dimensional Ising model the scalar curvature, $\mathcal{R}$, of this metric can be calculated explicitly in the thermodynamic limit and is found to be $\mathcal{R}=1+$ $\cosh (h) / \sqrt{\sinh ^{2}(h)+\exp (-4 \beta)}$. This is positive definite and, for physical fields and temperatures, diverges only at the zero-temperature, zero-field "critical point" of the model. In this note we calculate $\mathcal{R}$ for the one-dimensional $q$-state Potts model finding an expression of the form $\mathcal{R}=A(q, \beta, h)+B(q, \beta, h) / \sqrt{\eta(q, \beta, h)}$, where $\eta(q, \beta, h)$ is the Potts analogue of $\sinh ^{2}(h)+\exp (-4 \beta)$. This is no longer positive definite, but once again it diverges only at the critical point in the space of real parameters. We remark, however, that a naive analytic continuation to complex field reveals a further divergence in the Ising and Potts curvatures at the Lee-Yang edge.
\end{abstract}




\section{Introduction}

The notion of a distance between configurations in statistical-mechanical and lattice-field

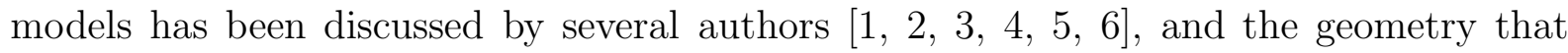
this endows on the manifold, $\mathcal{M}$, of the parameters characterising the models explored. This distance is measured using the Fisher-Rao metric [7], which is calculated from the Fisher information matrix for the system of interest. When we consider a spin model in field, $\mathcal{M}$ is a two-dimensional manifold parametrised by $\left(\theta^{1}, \theta^{2}\right)=(\beta, h)$. In this case, the components of the Fisher-Rao metric take the particularly simple form

$$
G_{i j}=-\partial_{i} \partial_{j} f,
$$

where $f$ is the reduced free energy per site and $\partial_{i}=\partial / \partial \theta^{i}$.

It has been suggested that in such a geometrization of statistical mechanics the scalar curvature, $\mathcal{R}$, of $\mathcal{M}$ plays a central role [1, 2, 3]. For a spin model in field, with the metric given in equ.(1), $\mathcal{R}$ may be calculated succinctly as

$$
\mathcal{R}=\frac{1}{2 G^{2}}\left|\begin{array}{lll}
\partial_{\beta}^{2} f & \partial_{\beta} \partial_{h} f & \partial_{h}^{2} f \\
\partial_{\beta}^{3} f & \partial_{\beta}^{2} \partial_{h} f & \partial_{\beta} \partial_{h}^{2} f \\
\partial_{\beta}^{2} \partial_{h} f & \partial_{\beta} \partial_{h}^{2} f & \partial_{h}^{3} f
\end{array}\right|,
$$

where $G=\operatorname{det}\left(G_{i j}\right)$. Since the only scale present near criticality for a model displaying a higher-order transition is the correlation length, $\xi$, it has been hypothesised on dimensional grounds that $\mathcal{R} \sim \xi^{d}$, where $d$ is the dimensionality of the system [1], 2, 3]. If we assume that hyperscaling holds, $\nu d=2-\alpha$, this leads to

$$
\mathcal{R} \sim|\xi|^{(2-\alpha) / \nu} .
$$

To test the behaviour of $\mathcal{R}$, one requires models which are solvable in field in order to obtain analytic expressions, and these are rather thin on the ground. Indeed, $\mathcal{R}$ has been calculated for the mean-field and Bethe-lattice Ising models 囮 and the above scaling behaviour verified. It has also been calculated for the one-dimensional Ising model [2] where it takes the remarkably simple form,

$$
\mathcal{R}_{\text {Ising }}=1+\frac{\cosh h}{\sqrt{\sinh ^{2} h+e^{-4 \beta}}} .
$$

In this case $\mathcal{R}$ is positive definite and diverges only at the zero-temperature, zero-field "critical point" of the model. The correlation length is given by

$$
\xi^{-1}=-\ln (\tanh (\beta)),
$$

so that $\xi \sim \exp (2 \beta)$ near criticality, and (3) holds there with $\alpha=1, \nu=1$ as expected $\left[\begin{array}{l}\text {. A } \\ \text {. A }\end{array}\right.$ second noteworthy feature of the Bethe-lattice and 1D Ising models concerns the metric associated with (1D). This metric is diagonalized using the corresponding renormalization group invariant.

\footnotetext{
${ }^{1}$ The Bethe lattice model also satisfies the postulated scaling, although there are some subtleties coming from the exponent $\alpha$ being zero 任.
} 
Given the shortage of explicitly calculable examples, any further additions to the list would be very worthwhile in order to see which features in the models are generic and which are particular to the models concerned. In this paper we discuss another example, the 1D $q$-state Potts model, where an expression for $\mathcal{R}$ may be obtained in a very similar manner to the Ising model (which is the $q=2$ case of the Potts model). We compare the properties of the curvature, $\mathcal{R}$, as well as the metric in the Potts model and the Ising model, highlighting both their structural similarities and differences in detail.

\section{The 1D Potts Model}

The partition function for the 1D $q$-state Potts model is given by

$$
Z_{N}(y, z)=\sum_{\{\sigma\}} \exp \left[\beta \sum_{j=1}^{N}\left(\delta\left(\sigma_{j}, \sigma_{j+1}\right)-\frac{1}{q}\right)+h \sum_{j=1}^{N}\left(\delta\left(\sigma_{j}, 1\right)-\frac{1}{q}\right)\right],
$$

where the spins, $\sigma_{j} \in\{1, \ldots, q\}$, are defined on the sites, $j \in\{1, \ldots N\}$, of the lattice and where we have defined $y=\exp (\beta)$ and $z=\exp (h)$ for later calculational convenience. The model may be solved by transfer matrix methods [8], just as the 1D Ising model. For general $q$ the full transfer matrix $T(y, z)$ may be written as $q-2$ diagonal elements, $(y-1)(y z)^{-1 / q}$, and a $2 \times 2$ factor $t(y, z)$ :

$$
t(y, z)=\frac{1}{(y z)^{1 / q}}\left(\begin{array}{cc}
y z & z^{1 / 2} \sqrt{q-1} \\
z^{1 / 2} \sqrt{q-1} & y+q-2
\end{array}\right) .
$$

The partition function is $Z_{N}(y, z)=\operatorname{Tr} T(y, z)^{N}$ and the eigenvalues of $T(y, z)$ are $\lambda_{0}, \lambda_{1}, \ldots, \lambda_{q-1}$, where

$$
\left.\begin{array}{l}
\lambda_{0} \\
\lambda_{1}
\end{array}\right\}=\frac{1}{2}\left[y(1+z)+q-2 \pm \sqrt{(y(1-z)+q-2)^{2}+(q-1) 4 z}\right](y z)^{-\frac{1}{q}}
$$

and $\lambda_{2}, \ldots \lambda_{q-1}=(y-1)(y z)^{-1 / q}$. The reduced free energy per site in the thermodynamic limit, $N \rightarrow \infty$, is thus given by $f=-\ln \lambda_{0}$.

It is straightforward to use this expression for the free energy in equ.(2) to obtain the curvature, $\mathcal{R}$. In the current notation we re-derive the expression for the Ising model $\mathbb{f}$ as

$$
\mathcal{R}_{\text {Ising }}=1+\frac{y(1+z)}{\sqrt{y^{2}-2 y^{2} z+y^{2} z^{2}+4 z}} .
$$

The expression for general $q$ is similar in form to this, and is

$$
\mathcal{R}_{\mathrm{Potts}}=A(q, y, z)+\frac{B(q, y, z)}{\sqrt{\eta(q, y, z)}},
$$

where the coefficients may be further broken down as $A(q, y, z)=\alpha(q, y, z) / \gamma(q, y, z)^{2}$ and $B(q, y, z)=\beta(q, y, z) / \gamma(q, y, z)^{2}$ and are smooth functions of $y$ and $z$ and do not diverge for finite (physical) temperature or field. Furthermore

$$
\eta(q, y, z)=[y(1-z)+q-2]^{2}+(q-1) 4 z .
$$

\footnotetext{
${ }^{2}$ There is a factor of two difference in the definitions of $\beta, h$ between the Ising and Potts notations coming from the different spin definitions.
} 
The expressions for $\alpha(q, y, z), \beta(q, y, z)$ and $\gamma(q, y, z)$ are very lengthy for general $q$ (although still easily obtained) so, for the sake of compactness, we write down only those for $q=3$, which already display the generic features. We have

$$
\begin{aligned}
& \alpha(3, y, z)=-\frac{1}{4}\left[-6-12 z+116 z^{2}+80 z^{3}-16 z^{4}\right. \\
& \quad+y\left(-44-68 z+400 z^{2}+552 z^{3}-192 z^{4}\right) \\
& \quad+y^{2}\left(-113-64 z+138 z^{2}+476 z^{3}-1004 z^{4}\right) \\
& \quad+y^{3}\left(-178+30 z+384 z^{2}-1884 z^{3}-2896 z^{4}+8 z^{5}\right) \\
& \quad+y^{4}\left(-188+458 z-222 z^{2}-276 z^{3}-3400 z^{4}+64 z^{5}\right) \\
& \quad+y^{5}\left(-106+592 z-474 z^{2}-68 z^{3}+1232 z^{4}+120 z^{5}\right) \\
& \quad+y^{6}\left(-17+294 z-368 z^{2}+250 z^{3}-127 z^{4}-32 z^{5}\right) \\
& \left.\quad+y^{7}\left(4+66 z-136 z^{2}+60 z^{3}+2 z^{5}+4 z^{4}\right)\right],
\end{aligned}
$$

and

$$
\begin{aligned}
& \beta(3, y, z)=-\frac{1}{4}\left[-30+12 z+468 z^{2}+768 z^{3}+240 z^{4}\right. \\
& \quad+y\left(-82-82 z+664 z^{2}+3604 z^{3}+1744 z^{4}-16 z^{5}\right) \\
& \quad+y^{2}\left(-171+12 z+1366 z^{2}+2292 z^{3}+1764 z^{4}-160 z^{5}\right) \\
& \quad+y^{3}\left(-261+213 z+1710 z^{2}+846 z^{3}-7584 z^{4}-756 z^{5}\right) \\
& \quad+y^{4}\left(-278+386 z+400 z^{2}-3804 z^{3}-8804 z^{4}-2488 z^{5}+8 z^{6}\right) \\
& \quad+y^{5}\left(-254+570 z-1094 z^{2}-5654 z^{3}-1584 z^{4}-3712 z^{5}+64 z^{6}\right) \\
& \quad+y^{6}\left(-165+504 z-1494 z^{2}-1008 z^{3}+891 z^{4}+1152 z^{5}+120 z^{6}\right) \\
& \quad+y^{7}\left(-51+263 z-866 z^{2}+986 z^{3}-203 z^{4}-97 z^{5}-32 z^{6}\right) \\
& \left.\quad+y^{8}\left(-4+66 z-182 z^{2}+188 z^{3}-72 z^{4}+2 z^{5}+2 z^{6}\right)\right],
\end{aligned}
$$

for the numerators, and

$$
\begin{aligned}
\gamma(3, y, z)= & -3-4 z-2 z^{2}-4 y-16 y z-16 y z^{2} \\
& -7 y^{2}+2 y^{2} z-31 y^{2} z^{2}-4 y^{3}+4 y^{3} z^{2},
\end{aligned}
$$

and

$$
\eta(3, y, z)=1+8 z+2 y-2 y z+y^{2}-2 y^{2} z+y^{2} z^{2},
$$

for the terms in the denominators.

In zero-field $(z=1)$ the expression for $\mathcal{R}$ is much more compact and is written for general $q$ as

$$
\mathcal{R}=\frac{(y+q-1)\left(4 y^{2}+(q-2) y-(q-2)(q-1)\right)}{(q-1)(2 y+q-2)^{2}} .
$$

We see that as $y$ ranges from 1 to $\infty, \mathcal{R}$ ranges from $(4-q) /(q-1)$ to $\infty$. In particular, the sign of the $y=1(\beta=0)$ limit of $\mathcal{R}$ changes at $q=4$, although the general morphology of $\mathcal{R}$ as a function of $y$ and $z$ remains the same for all $q>2$ as we see below. 
The correlation length for the one-dimensional Potts model is defined in a similar manner to that of the Ising model,

$$
\xi^{-1}=-\ln \left(\frac{\lambda_{1}}{\lambda_{0}}\right),
$$

so $\xi \sim y$ for $z=1, y \rightarrow \infty$. We thus retrieve the expected scaling of $\mathcal{R}$ for the onedimensional Potts model from equ.(3), namely $\mathcal{R} \sim y$ as $y \rightarrow \infty$. The exponents, as for the Ising model, are $\alpha=1, \nu=1$.

The general features of $\mathcal{R}$ at non-zero temperature and field are perhaps easiest seen in a contour plot as a function of $y$ and $z$. In Fig.1 we show the Ising $(q=2)$ case which has certain non-generic features. The $\pm h$ symmetry of the Ising model is manifest as a $z \rightarrow 1 / z$ symmetry in the plot of $\mathcal{R}$. In addition, one can see that $\mathcal{R}$ is positive for all $y$ and $z$. The maximum of $\mathcal{R}$ for a given $y$ value lies along the zero field line at $z=1$.

In Fig.2, $\mathcal{R}$ is plotted for the 3-state Potts model, using the expressions given above for $A(3, y, z)$ and $B(3, y, z)$. We see that there is no longer a $z \rightarrow 1 / z$ symmetry and that $\mathcal{R}$ is not positive definite. This behaviour is typical of the $q>3$ case, as can also be seen from the $q=10$ results plotted in Fig.3. It is also clear from Fig.3. that the $z=1, y=1$ limit is negative unlike the 3 -state model, as indicated by equ.(16). When $q>2$ the maximum of $\mathcal{R}$ no longer lies on the zero-field line though its locus is still simply determined, as we discuss in the next section.

\section{Remarks on Co-ordinates and Geodesics}

We have concentrated so far on the curvature $\mathcal{R}$, which is of course a geometric invariant and independent of the particular co-ordinate scheme we use in the calculation. The properties of the particular metrics and line elements used in the calculation are also of some interest [5]. The metric for the 1D Ising model calculated from equ.(11) gives the line element

$$
\begin{aligned}
d s^{2} & =\frac{\mathrm{e}^{-4 \beta}}{\left(\sinh ^{2} h+\mathrm{e}^{-4 \beta}\right)^{3 / 2}} \times\left[\frac{4 \mathrm{e}^{-4 \beta} \cosh h+8 \sinh ^{2} h\left(\cosh h+\sqrt{\sinh ^{2} h+\mathrm{e}^{-4 \beta}}\right)}{\left(\cosh h+\sqrt{\sinh ^{2} h+\mathrm{e}^{-4 \beta}}\right)^{2}} d \beta^{2}\right. \\
& \left.+4 \sinh h d \beta d h+\cosh h d h^{2}\right] .
\end{aligned}
$$

Although this is clearly non-diagonal, the choice of $\beta$ and $h$ as co-ordinates has the advantage of giving the simplified expression, equ.(2), for $\mathcal{R}$ since they appear as linear multipliers in the Hamiltonian.

The metric can be diagonalized by inspection in this case, and this preferred choice of co-ordinates turns out to be rather interesting from the physical point of view. If one considers a decimation type renormalization transformation on a 1D Ising chain (or ring) where every other vertex is decimated and the length scale suitably adjusted, then one can exactly determine the relations between the renormalized parameters $\beta^{\prime}, h^{\prime}$ and the originals $\beta, h$ from

$$
Z_{N / 2}\left(\beta^{\prime}, h^{\prime}\right)=A^{N} Z_{N}(\beta, h)
$$

where $Z_{N / 2}$ is the decimated partition function and $Z_{N}(\beta, h)$ is the original one. In addition to the parameter transformation there is also an unimportant overall scaling 
factor, $A^{N}$. This transformation has an invariant $\rho=\mathrm{e}^{2 \beta} \sinh h$, which is in effect encoding the invariance of the magnetization under the transformation since

$$
M=\frac{\rho}{\sqrt{1+\rho^{2}}} .
$$

If the co-ordinates are transformed from $\beta, h$ to $\beta, \rho$ then, using

$$
d \rho=2 \mathrm{e}^{2 \beta} \sinh h d \beta+\mathrm{e}^{2 \beta} \cosh h d h,
$$

one finds the diagonalized expression

$$
d s^{2}=\frac{1}{\sqrt{\left(1+\rho^{2}\right)\left(\mathrm{e}^{4 \beta}+\rho^{2}\right)}}\left[\frac{4 \mathrm{e}^{4 \beta} d \beta^{2}}{\left(\sqrt{1+\rho^{2}}+\sqrt{\mathrm{e}^{4 \beta}+\rho^{2}}\right)^{2}}+\frac{d \rho^{2}}{\left(1+\rho^{2}\right)}\right] .
$$

The use of the renormalization group invariant, or alternatively the magnetization, also diagonalizes the metric for the Ising model on a Bethe lattice. It is therefore natural to ask whether this feature persists in the Potts models considered here. Although the Potts metric and line elements are rather more complicated than those for the Ising model, the same general structure is once again apparent

$$
\begin{aligned}
d s^{2} & =\frac{(q-1)}{\eta(q, y, z)^{(3 / 2)}} \times\left[\frac{C(q, y, z)+D(q, y, z) \sqrt{\eta(q, y, z)}}{\tilde{\lambda}(q, y, z)^{2}} d \beta^{2}+4[y z(z-1)] d \beta d h\right. \\
& \left.+z[y(1+z)+q-2] d h^{2}\right]
\end{aligned}
$$

where $\eta(q, y, z)$ is defined in equ.(11) and

$$
\tilde{\lambda}(q, y, z)=y(1+z)+q-2+\sqrt{(y(1-z)+q-2)^{2}+(q-1) 4 z}
$$

is proportional to $\lambda_{0}$, the dominant eigenvalue. The functions $C(q, y, z)$ and $D(q, y, z)$ are both easily calculable, but rather long, and are not reproduced here.

If we carry out a decimation on the $1 \mathrm{D}$ Potts model

$$
Z_{N / 2}\left(y^{\prime}, z^{\prime}\right)=A^{N} Z_{N}(y, z)
$$

then the invariant is given by

$$
\tilde{\rho}=\frac{[y(1-z)+q-2]}{\sqrt{z}},
$$

which is again related to the magnetization. Following the Ising procedure, we can change variables $\beta, h$ to $\beta, \tilde{\rho}$ using

$$
d \tilde{\rho}=\frac{y(1-z)}{\sqrt{z}} d \beta-\frac{1}{2} \frac{[y(1+z)+q-2]}{\sqrt{z}} d h
$$

and find that the metric is, indeed, diagonalized.

In the Ising model it can be shown that the line $\rho=0$ (i.e. $h=0$ or $z=1$ ) is a geodesic of the metric (11). In Fig.1 this is the line running along the ridge of $\mathcal{R}$. For 
$q \neq 2$ Potts models the local maximum in $\mathcal{R}$ no longer lies at $z=1$, as is clear from Fig.2 and Fig.3, however an analysis of the geodesic equations

$$
\begin{aligned}
& \frac{d V^{\beta}}{d s}+\Gamma_{\beta \beta}^{\beta} V^{\beta} V^{\beta}+2 \Gamma_{\beta h}^{\beta} V^{\beta} V^{h}+\Gamma_{h h}^{\beta} V^{h} V^{h}=\lambda(s) V^{\beta} \\
& \frac{d V^{h}}{d s}+\Gamma_{\beta \beta}^{h} V^{\beta} V^{\beta}+2 \Gamma_{\beta h}^{h} V^{\beta} V^{h}+\Gamma_{h h}^{h} V^{h} V^{h}=\lambda(s) V^{h}
\end{aligned}
$$

where $s$ parameterizes the flow lines, $V^{\beta}=d \beta / d s, V^{h}=d h / d s$, the $\Gamma$ are the various Christoffel symbols for the metric of equ.(1) , and $\lambda(s)$ allows for non-affine parameters, shows that the line $z=1$ is still a geodesic. To prove this consider a vector field with a flow line along $z=1(h=0)$. This flow line has $V^{h}=0$ so equations (28) become

$$
\begin{aligned}
\frac{d V^{\beta}}{d s}+ & \left.\left(\Gamma_{\beta \beta}^{\beta}\right)\right|_{h=0} V^{\beta} V^{\beta}=\lambda(s) V^{\beta} \\
& \left.\left(\Gamma_{\beta \beta}^{h}\right)\right|_{h=0} V^{\beta} V^{\beta}=0 .
\end{aligned}
$$

The first of these equations is a second order ordinary differential equation for $\beta(s)$ which always has a solution. The second requires $\left.\left(\Gamma_{\beta \beta}^{h}\right)\right|_{h=0}=0$ and it can easily be shown, using the metric (23), that this is indeed the case. Hence the line $z=1$ is a geodesic of the metric (23) for any value of $q$.

A different change of variable in the Potts models brings one back to something very similar to the Ising picture. If we transform $z$ to

$$
w=\frac{y z}{y+q-2}
$$

and leave $y$ untouched, we find that the local maximum in $\mathcal{R}$ lies on the line $w=1$. We show $\mathcal{R}$ plotted against $w$ in Fig.4 for the 3-state Potts model for comparison with Fig.2. The picture is similar for higher $q$. This transformation does not greatly simplify $\alpha(q, y, z), \beta(q, y, z)$ or $\gamma(q, y, z)$ and we do not reproduce the expressions here.

In summary, although the choice of $\beta, h$ as parameters is natural in any calculation of $\mathcal{R}$, the metric is diagonalized for both the $1 \mathrm{D}$ Ising and Potts models when the renormalization group invariant, or equivalently the magnetization, is used as a co-ordinate instead of $h$. The line $z=1$ is a geodesic of the metric (1) for any $q$.

\section{A Lee-Yang Divergence}

Some years ago Lee and Yang [9] addressed the question of how the singularities associated with field-driven phase transitions in Ising-like spin models on lattices arose in the thermodynamic limit. This was later extended by various authors to other models and to temperature-driven transitions [10, 11]. Lee and Yang observed that the zeroes of the partition function for a spin model in a complex external field on a finite lattice would give rise to singularities in the free energy. In the thermodynamic limit these complex zeroes move in to pinch the real axis, signalling the the onset of a physical phase transition. Typically, the loci of zeroes are lines in the complex field or temperature plane and when the endpoints of such lines occur at non-physical (i.e. complex) external field values they 
can be considered as ordinary critical points with an associated edge critical exponent, usually dubbed the Lee-Yang edge exponent [10].

The Lee-Yang zeroes for the one-dimensional Potts model on a periodic chain with $N$ sites are given by the solutions [8, 12] of

$$
Z_{N}=\left(\lambda_{1}\right)^{N}+\left(\lambda_{0}\right)^{N}=0 \quad \Leftrightarrow \quad \lambda_{1}=\exp \left(\frac{i n \pi}{N}\right) \lambda_{0}
$$

where $\lambda_{0,1}$ are the eigenvalues given in equ. (8) and $n$ is odd. In the thermodynamic limit the locus of zeroes is determined by $\left|\lambda_{0}\right|=\left|\lambda_{1}\right|$ or

$$
\eta(q, y, z)=[y(1-z)+q-2]^{2}+(q-1) 4 z=0
$$

which can be satisfied for complex (in the $q=2$ Ising case, purely imaginary) values of $h$.

Unlike the Ising model case, the Lee-Yang zeroes for the 1D $q \neq 2$ Potts models do not lie on the unit circle in the complex $z$ plane. However, precisely the change of co-ordinates we used in discussing the Potts metrics in the previous section, $w=y z /(y+q-2)$, places them on the unit circle in the complex $w$ plane. If we associate a "field" with $w$ via $w=\exp (\tilde{h})$, then purely imaginary values of $\tilde{h}$ give the zeroes, as in the Ising case ${ }^{3}$.

From these considerations, it is clear that $\mathcal{R}$ will also diverge as the locus of zeroes is approached for both Ising and Potts models if we allow ourselves the liberty of an analytic continuation of the field to complex $h$ values once $\mathcal{R}$ has been calculated, since $\mathcal{R}=A+B / \sqrt{\eta}$ and $A, B$ are finite as $\eta \rightarrow 0$. The presence of the square root means that the divergence is characterised by an exponent $\sigma=-1 / 2$ which is the Lee-Yang edge exponent for the one-dimensional Potts (and Ising) model [10].

The status of these observations are a little unclear to us, since the calculation of $\mathcal{R}$ has assumed a real metric geometry throughout and such an arbitrary continuation in the final expression might be rather dangerous. One is on slightly firmer ground with the Ising model, since in that case the required continuation is to purely imaginary fields which correspond simply to a change in the signature of the metric in $\beta, \rho$ co-ordinates. The use of the $w$ co-ordinate in the Potts case does, however, suggest a similar interpretation since there the zeroes occur for imaginary values of $\tilde{h}$. With these caveats, it is nonetheless interesting that the Lee-Yang edge transition is still visible as a divergence in $\mathcal{R}$.

\section{Conclusions}

We have seen that the scalar curvature, $\mathcal{R}$, of the Fisher-Rao metric may be obtained for the 1D $q$-state Potts model in a very similar fashion to the 1D Ising model since the free energy can be calculated in field in both cases using transfer matrix methods.

Although $\mathcal{R}$ for the $q$-state Potts model cannot be expressed as succinctly as for the Ising model it still has the same general form, $\mathcal{R}=A+B / \sqrt{\eta}$, and diverges only at the zero temperature critical point of the Potts model for physical temperature and field values, i.e. $y=1 \ldots \infty$ and $z=0 \ldots \infty$. We wrote down $\mathcal{R}$ explicitly here for $q=3$, but noted it was a simple matter to calculate it for arbitrary $q$. We observed that there were some features of the Ising model $\mathcal{R}$ which did not persist for general $q$, as was clear from

\footnotetext{
${ }^{3}$ This is not, of course, the field $h$ appearing in the Hamiltonian.
} 
contour plots. In particular, $\mathcal{R}$ was no longer positive definite and the $z \rightarrow 1 / z$ symmetry of the Ising model was no longer present.

The choice of co-ordinate scheme used in the calculation of $\mathcal{R}$ was also discussed. We noted that although $\beta, h$ were a natural choice from the point of view of simplifying this calculation, the use of the renormalization group invariant in place of $h$ diagonalized the metric for both the Ising and Potts models. A different choice of coordinates, involving a rescaling of $z$ was also employed to identify the local maximum in $\mathcal{R}$. For any value of $q$ the line $z=1(h=0)$ is a geodesic of the Fisher-Rao metric. Finally, we observed that if complex field values are permitted there is a divergence in $\mathcal{R}$ at the Lee-Yang edge with an exponent $\sigma=-1 / 2$.

We have confined our discussion here to physical values of $q$, i.e. $2,3, \ldots$, in the Potts models. Since $q$ appears as a parameter in the transfer matrix solution there is in principle no barrier to extending it to general non-integer $q$ and also to $q<2$. The $q \rightarrow 1$ limit of the Potts model is related to percolation, for example, so the behaviour of $\mathcal{R}$ might be of interest in this limit too. It would also be interesting to calculate $\mathcal{R}$ for models with genuine transitions at finite $\beta$, an exercise that has so far been confined to mean-field or mean-field-like spin models. Possibilities which suggest themselves in this context are the Ising model on planar random graphs [13] and the spherical model.

\section{Acknowledgements}

D.J. was partially supported by EC IHP network "Discrete Random Geometries: From Solid State Physics to Quantum Gravity" HPRN-CT-1999-000161. D.J. and R.K. were also partially supported by an Enterprise Ireland/British Council Research Visits Scheme grant.

\section{References}

[1] G. Ruppeiner, Rev. Mod. Phys. 67 (1995) 605; Phys. Rev. A 20 (1979) 1608; ibid A 24 (1980) 488.

[2] H. Janyszek and R. Mrugała, Phys. Rev. A 39 (1989) 6515;

H. Janyszek, Rep. Math. Phys. 24 (1986) 1; ibid 11.

[3] D. Brody and N. Rivier, Phys. Rev. E 51, 1006 (1995);

D. Brody and A. Ritz, Nucl. Phys. B 522 (1998) 588;

E.J. Brody, Phys. Rev. Lett. 58 (1987) 179;

D. Brody and L. Hughston, Proc. R. Soc. London A 455 (1999) 1683.

[4] B. Dolan, Proc. Roy. Soc. Lond. A 454 (1998) 2655.

[5] B. Dolan, Int. J. Mod. Phys. A 12 (1997) 2413.

[6] B. Dolan, Int. J. Mod. Phys. A 10 (1995) 2439; ibid 2703; Nucl. Phys. B 528 (1998) 553 ;

D. O'Connor and C.R. Stephens, Prog. Theor. Phys. 90 (1993) 747; 
J. Gaite and D. O'Connor, Phys. Rev. D 54 (1996) 5163;

A.C. Irving and J.C. Sexton, Phys. Rev. D 55 (1997) 5456.

[7] R.A. Fisher, Phil. Trans. R. Soc. Lond. 222 (1922) 309;

C.R. Rao, Bull. Calcutta Math. Soc. 37 (1945) 81.

[8] Z. Glumac and K. Uzelac, J. Phys. A 27 (1994) 7709.

[9] T.D. Lee and C.N. Yang, Phys. Rev. 87 (1952) 410; C.N. Yang and T.D. Lee, Phys. Rev. 87 (1952) 404.

[10] J. Lebowitz and O. Penrose, Comm. Math. Phys. 11 (1968) 99;

G. Baker, Phys. Rev. Lett. 20 (1968) 990;

R. Abe, Prog Theor. Phys. 37 (1967) 1070; ibid 38 (1967) 72; ibid 38 (1967) 568;

S. Ono, Y. Karaki, M. Suzuki and C. Kawabata, J. Phys. Soc. Japan 25 (1968) 54;

D. Gaunt and G. Baker, Phys. Rev. B 1 (1970) 1184;

P. Kortman and R. Griffiths, Phys. Rev. Lett. 27 (1971) 1439;

M. Fisher, Phys. Rev. Lett. 40 (1978) 1611.

[11] M. Fisher, in "Lectures in Theoretical Physics" VII C (University of Colorado Press, Boulder, 1965).

[12] B. Dolan, Phys. Rev E 52 (1995) 4512; Erratum-ibid E 53 (1996) 6590.

[13] V.A. Kazakov, Phys. Lett. A 119 (1986) 140;

D.V. Boulatov and V.A. Kazakov, Phys. Lett. B 186 (1987) 379. 


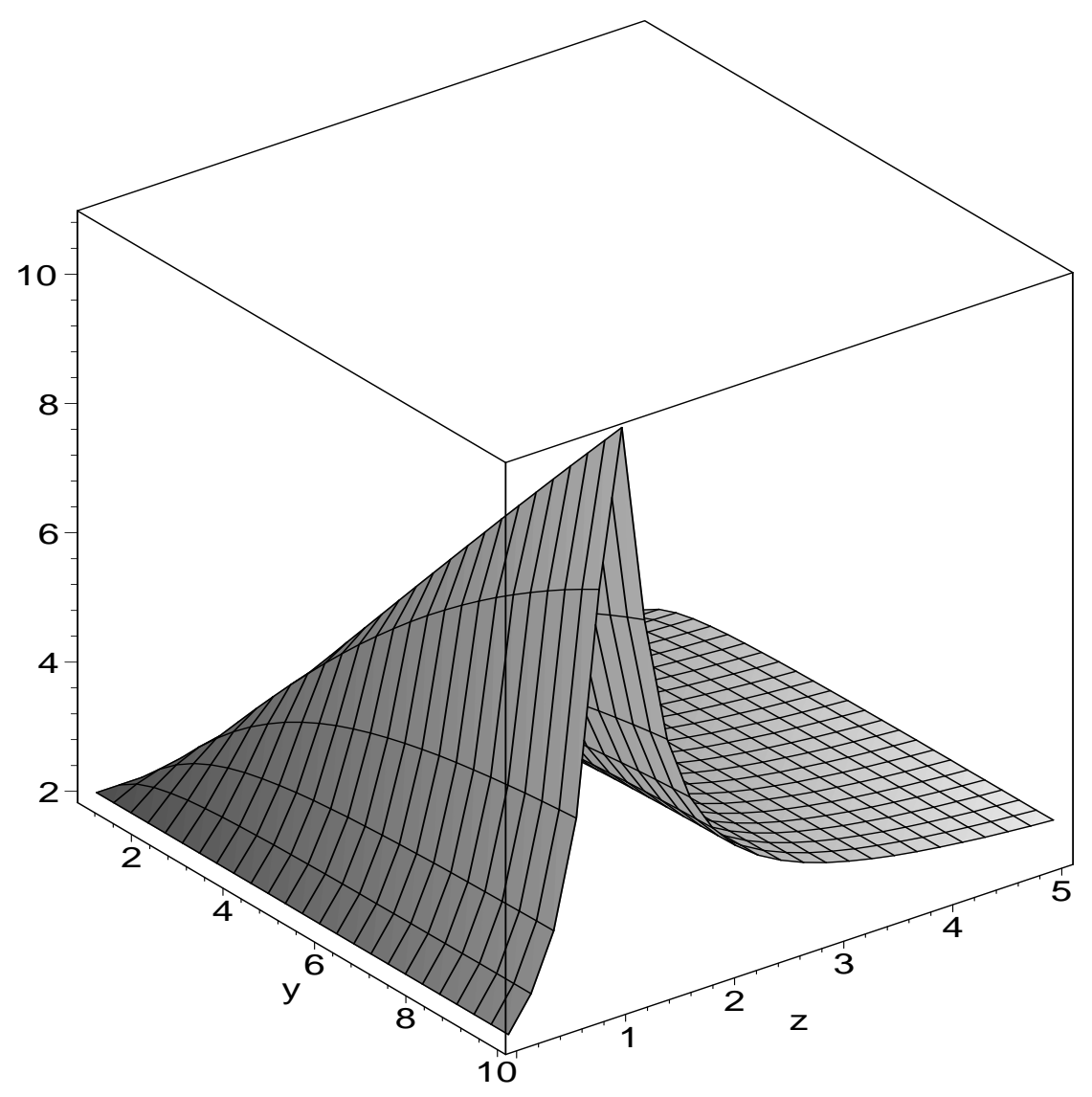

Figure 1: A plot of $\mathcal{R}$ for the Ising model for $y=1 \ldots 10, z=0 \ldots 5$. The positivity of $\mathcal{R}$ and the expected $z \rightarrow 1 / z$ symmetry are both apparent. 


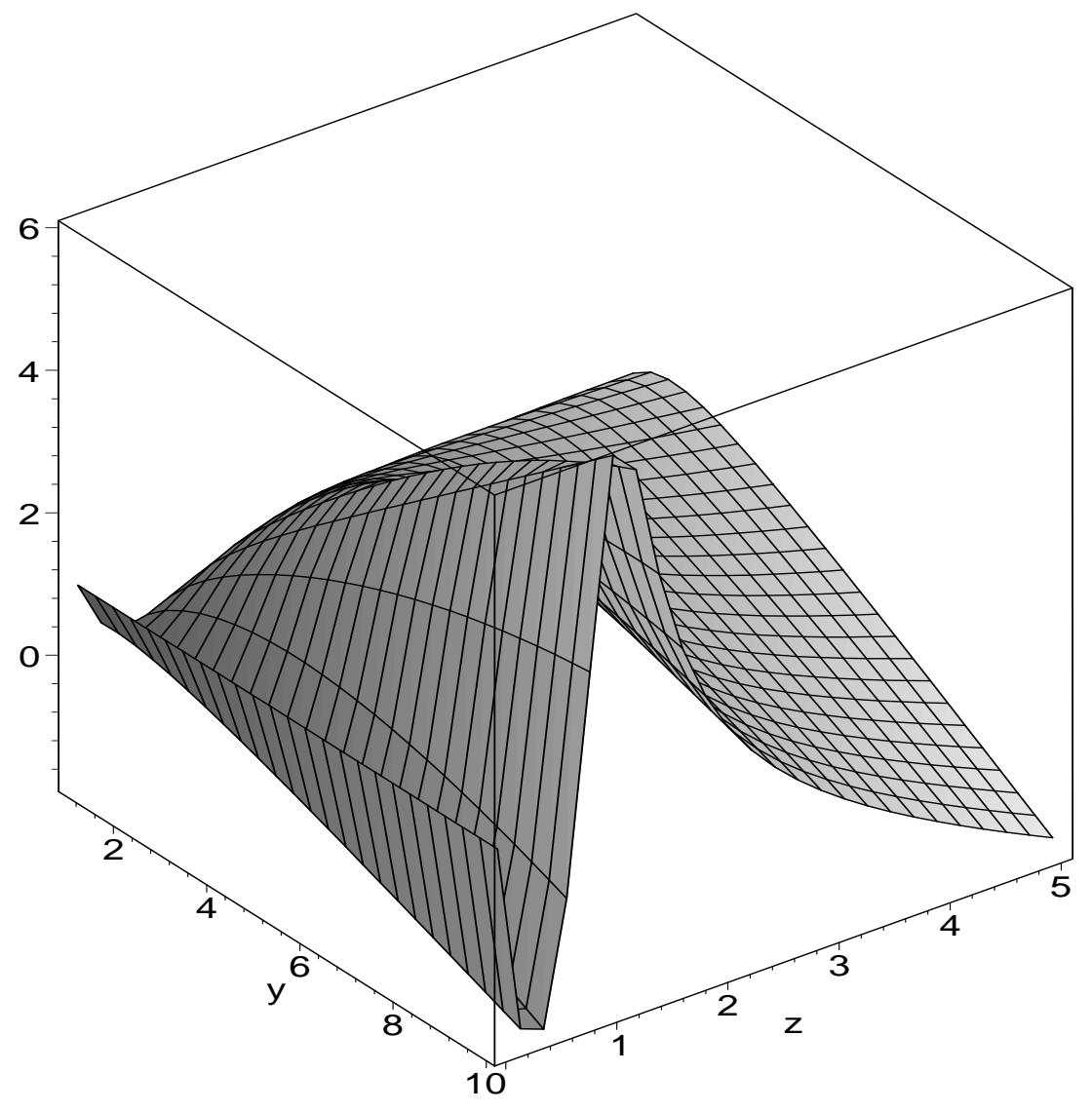

Figure 2: A plot of $\mathcal{R}$ for the 3-state Potts model for $y=1 \ldots 10, z=0 \ldots 5$. $\mathcal{R}$ is no longer positive definite for physical values of $y, z$ and there is no $z \rightarrow 1 / z$ symmetry. In addition the maximum of $\mathcal{R}$ does not lie at $z=1$, though this is perhaps clearer for higher $q$. 


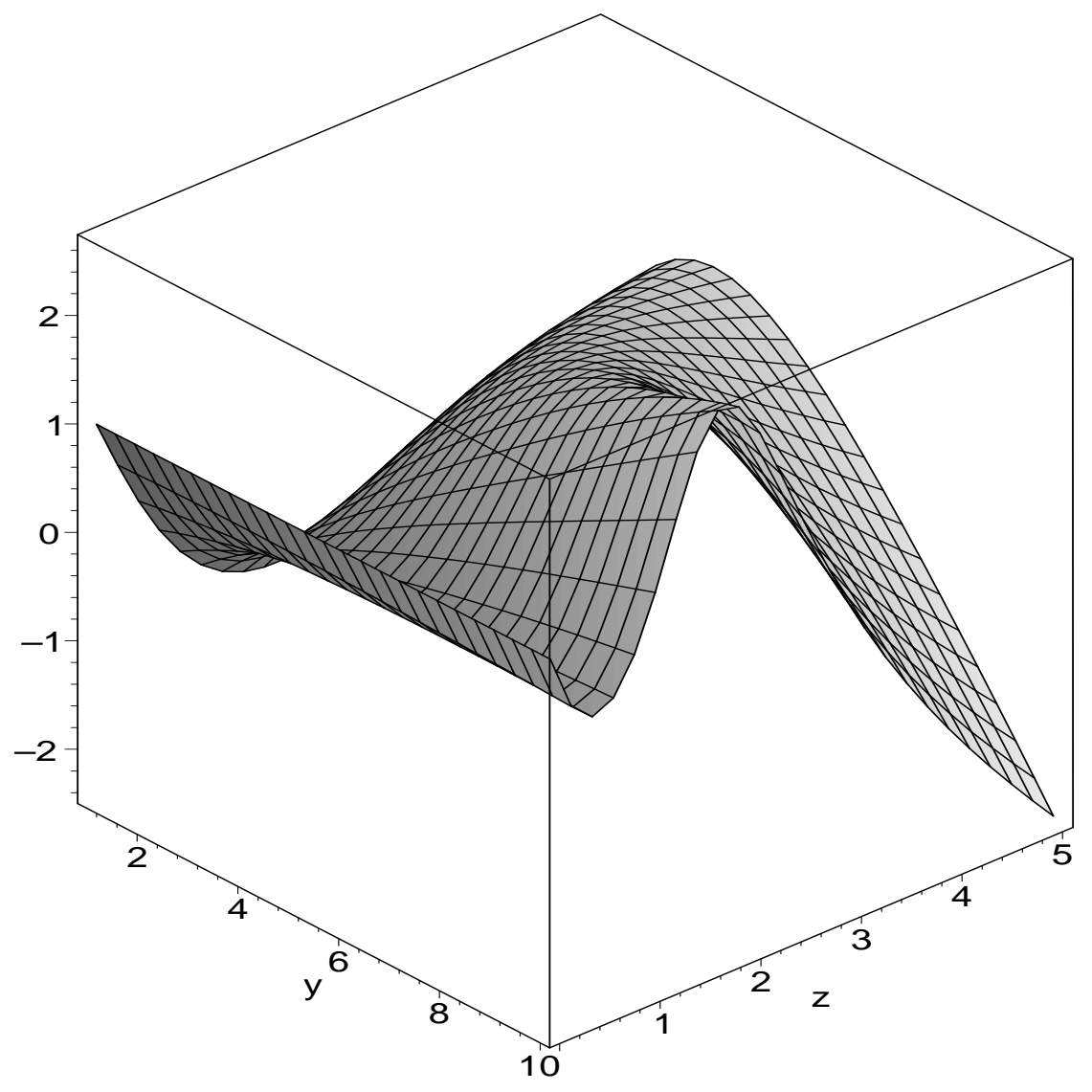

Figure 3: A plot of $\mathcal{R}$ for the 10 -state Potts model for $y=1 \ldots 10, z=0 \ldots 5$. In this case it is quite clear that the maximum of $\mathcal{R}$ does not lie at $z=1$. 


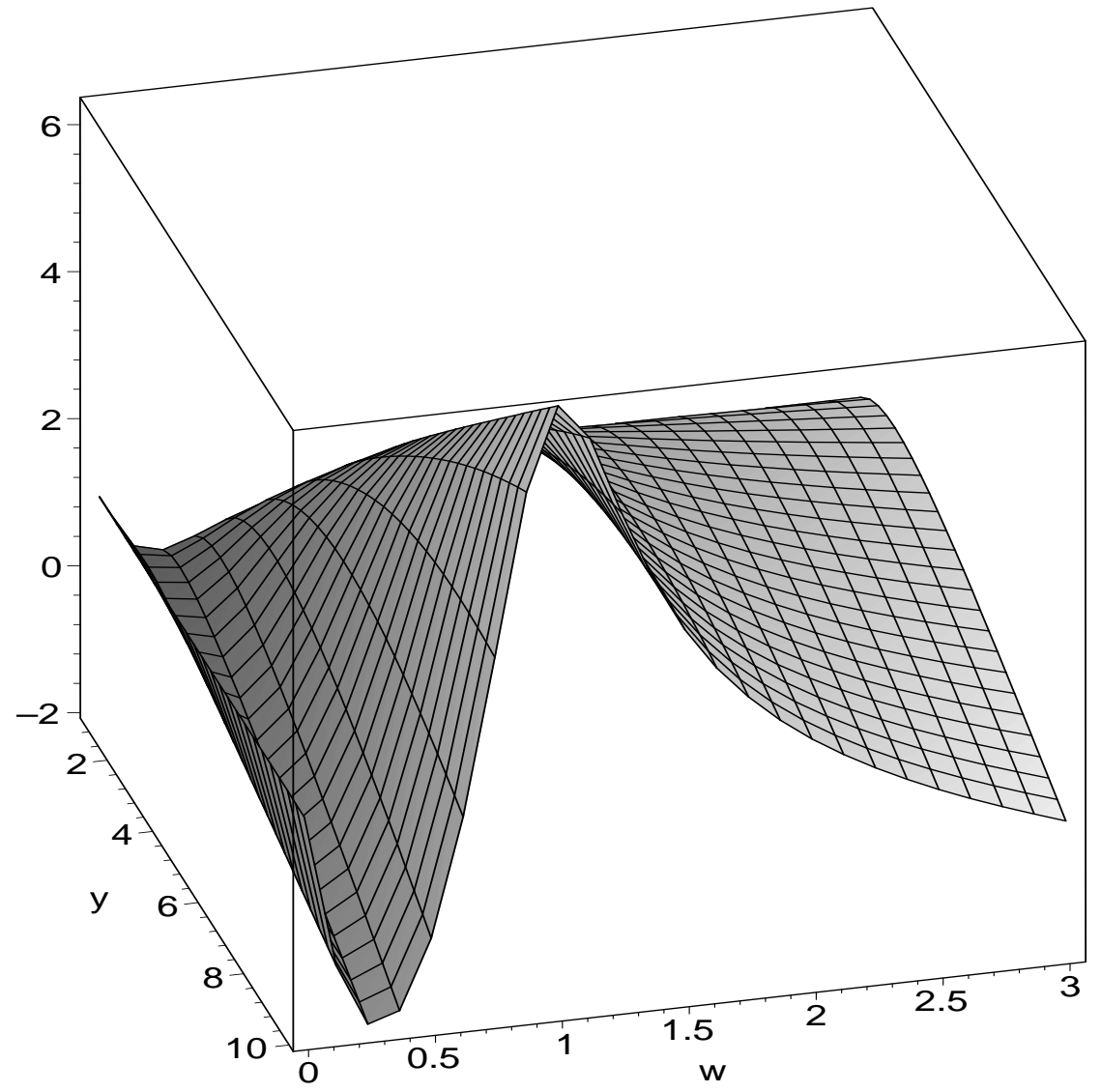

Figure 4: A plot of $\mathcal{R}$ for the 3-state Potts model for $y=1 \ldots 10, w=0 \ldots 3$. Note that the maximum of $\mathcal{R}$ lies at $w=1$ 